\title{
A Genome-Wide Search for Bipolar Disorder Risk Loci Modified by Mitochondrial Genome Variation
}

\author{
Euijung Ryu $^{a}$ Malik Nassan $^{b}$ Gregory D. Jenkins ${ }^{a}$ Sebastian M. Armasu ${ }^{a}$ \\ Ana Andreazza $^{f}$ Susan L. McElroy ${ }^{c, d}$ Marquis P. Vawter ${ }^{e}$ Mark A. Frye $^{b}$ \\ Joanna M. Biernackaa, b \\ Departments of a Health Sciences Research and ${ }^{b}$ Psychiatry and Psychology, Mayo Clinic, Rochester, MN, \\ 'Lindner Center of HOPE, Mason, $\mathrm{OH}$, dUniversity of Cincinnati College of Medicine, Cincinnati, $\mathrm{OH}$, and \\ e Psychiatry and Human Behavior, School of Medicine, University of California, Irvine, Irvine, CA, USA; \\ fDepartment of Psychiatry and Pharmacology, University of Toronto, Toronto, ON, Canada
}

\section{Keywords}

Mitochondrial genome $\cdot$ Bipolar disorder .

Genome-wide association study · Interaction .

Single-nucleotide polymorphism

\begin{abstract}
Mitochondrial DNA mutations have been reported to be associated with bipolar disorder (BD). In this study, we performed genome-wide analyses to assess mitochondrial single-nucleotide polymorphism (mtSNP) effects on BD risk and early-onset $\mathrm{BD}$ (EOBD) among BD patients, focusing on interaction effects between nuclear SNPs (nSNPs) and mtSNPs. Common nSNP and mtSNP data from European American BD cases $(n=1,001)$ and controls $(n=1,034)$ from the Genetic Association Information Network BD study were analyzed to assess the joint effect of nSNP and nSNP-mtSNP interaction on the risk of $B D$ and EOBD. The effect of nSNPmtSNP interactions was also assessed. For BD risk, the
\end{abstract}

strongest evidence of an association was obtained for nSNP rs1880924 in MGAM and mtSNP rs3088309 in CytB $\left(p_{\text {joint }}=\right.$ $8.2 \times 10^{-8}, p_{\text {int }}=1.4 \times 10^{-4}$ ). Our results also suggest that the minor allele of the nSNP rs583990 in CTNNA2 increases the risk of EOBD among carriers of the mtSNP rs3088309 minor allele, while the nSNP has no effect among those carrying the mtSNP major allele $\left(\mathrm{OR}=4.53\right.$ vs. $1.05, p_{\text {joint }}=2.1 \times 10^{-7}$, $\left.p_{\text {int }}=1.16 \times 10^{-6}\right)$. While our results are not statistically significant after multiple testing correction and a large-sample replication is required, our exploratory study demonstrates the potential importance of considering the mitochondrial genome for identifying genetic factors associated with $\mathrm{BD}$.

(c) 2017 S. Karger AG, Basel

Euijung Ryu and Malik Nassan contributed equally to this work. This paper was presented in part at the Annual Meeting of the International Society of Bipolar Disorder and the Biannual Meeting of the International Society of Affective Disorders in Amsterdam, The Netherlands, in July of 2016.

\section{KARGER}

(C) 2017 S. Karger AG, Basel

E-Mail karger@karger.com

www.karger.com/mnp
Euijung Ryu, $\mathrm{PhD}$ Mayo Clinic 200 First Street SW

Rochester, MN 55905 (USA)

E-Mail Ryu.Euijung@mayo.edu 


\section{Introduction}

Bipolar disorder (BD) is a serious and persistent mental illness characterized by recurrent episodes of mania/ hypomania and depression $[1,2]$. Among all mental and neurological disorders, BD was ranked fourth as a cause of global burden based on disability-adjusted life years [3]. Many genetic studies, including both candidate gene studies and genome-wide association studies (GWAS), have been performed to identify BD susceptibility genes. By searching the nuclear genome, GWAS of large samples have identified multiple loci associated with the risk of $\mathrm{BD}$, including CACNA1C, ANK3, ODZ4, SYNE1, and TRANK1 $[4,5]$. However, genetic variants from these loci only explain a small portion of BD heritability [6].

Mitochondrial dysfunction has been implicated in $\mathrm{BD}$ [7-10]. The energy dysregulation has been characterized by increased reactive oxygen species production, decreased mitochondrial complex subunits in the brain, ATP-dependent proteasome degradation, and an increase in lactate with a corresponding decreased intracellular $\mathrm{pH}$ [11-16]. Because mitochondrial DNA (mtDNA) encodes a number of mitochondrial proteins, it has been hypothesized that inherited variation in the mitochondrial genome may affect mitochondrial dysfunction and thus BD risk. Consistent with this hypothesis, clinical studies have found that subjects with maternal relatives with $\mathrm{BD}$ have a higher disease risk than those with paternal relatives with $\mathrm{BD}$, supporting a potential maternal mtDNA transmission of risk [17]. In addition, a recent study based on induced pluripotent stem cell lines showed that mtDNA genes were differently expressed in young $\mathrm{BD}$ hippocampal neurons compared to normal neurons and in lithium-responsive BD neurons with and without lithium treatment [18]. Research on the role of the mitochondrial genome in BD risk has revealed candidate associations with mitochondrial point mutations, deletions, haplogroups (subjects sharing the same maternal ancestral haplotypes), and copy number variations [1922 ]. For instance, the rare mtDNA mutation $3644 \mathrm{~T}>\mathrm{C}$ was found to be associated with BD [7, 19]. In addition, various mitochondrial single-nucleotide polymorphisms (mtSNPs) have been proposed to be associated with BD [19, 22-24]. For example, the 10398A variant was significantly associated with the risk of $\mathrm{BD}$ and a better response to lithium, as well as impaired prefrontal glucose utilizations $[23,25,26]$. MtDNA deletion (4,977-bp deletion), known as "the common deletion," was shown to be overrepresented in $\mathrm{BD}$ brain tissue compared to controls, in particular in the dorsolateral prefrontal cortex [21]. With regard to mitochondrial haplogroups, overrepresentation of N9a in BD patients has been reported [22]. In addition, some haplogroups (U, K, and Uk) showed significantly lower cerebellar $\mathrm{pH}$, leading to speculation that $\mathrm{pH}$ variation in the brain could be inherited through mtDNA and constitute a risk factor for $\mathrm{BD}$. A decrease in mtDNA copy numbers has also been observed in brains of BD subjects [27]. These studies suggest that mitochondrial genome variation plays a role in $\mathrm{BD}$; however, they are mainly small studies without adequate multiple testing correction and no replication, and thus further research is needed to determine the impact of mtDNA variation on the risk of $\mathrm{BD}$ and related phenotypes.

It is also important to note that mitochondrial proteins are encoded by both nuclear and mtDNA, with the vast majority of genes encoding mitochondrial proteins being products of nuclear DNA (nDNA). In fact, MitoCarta, which is an inventory of genes encoding proteins with strong support of mitochondrial localization, includes more than 1,000 genes encoded by the human nuclear genome [28]. The products of these nuclear and mitochondrial genes work together to control transcription and translation of mitochondrial genes and to form the complexes of the respiratory chain that carry out oxidative phosphorylation and energy production. Therefore, genetic variations from both mtDNA and nDNA may affect $\mathrm{BD}$ risk by influencing mitochondrial function. While further investigation of the role of mitochondrion-related genes in $\mathrm{BD}$ is needed, variation in certain mitochondrionrelated nDNA genes has been reported to be associated with BD. For example, DISC-1, which encodes a protein involved in mitochondrial dynamics and trafficking, was identified as a potential susceptibility gene for schizophrenia and BD in a large Scottish pedigree [29, 30]. Studies have also suggested that genetic variation in the promoter region of NDUFV2, a mitochondrial complex I gene, is associated with $\mathrm{BD}[31,32]$. A potential role of this gene in BD was also supported by the observed downregulation of NDUFV2 in lymphoblastoid cell lines from patients with BD type I but not BD type II [33]. While the association of $\mathrm{BD}$ with nDNA-encoded mitochondrial genes is not yet well established, it warrants further investigation.

Many commonly used genome-wide genotyping platforms include mtSNP content and thus many existing GWAS datasets contain mtSNP data. However, mtSNPs have typically been excluded from complex-trait genetic risk discovery efforts, and studies incorporating both nuclear and mitochondrial genome data are extremely rare [34]. Exceptions include a study of outcomes following traumatic brain injury (interaction between nuclear gene 
$A P O E$ and mitochondrial haplogroup $\mathrm{K}$ ), a study of lateonset Alzheimer's disease (interaction between nuclear variant $A P O E 4+$ status and mitochondrial subhaplogroup H5), and an ulcerative colitis study (mtSNP A 10550G being an independent risk factor from nucleusencoded susceptibility loci) [34-36].

In $\mathrm{BD}$, a recent GWAS using the Genetic Association Information Network (GAIN) BD dataset reported that 2 mtSNPs in ND1 (rs28357968) and CytB (rs28357375) showed a nominal association (without correcting for multiple testing) with the risk of $\mathrm{BD}$ [37]. However, no prior studies in $\mathrm{BD}$ have investigated potential interaction effects of genetic variations from both genomes despite the fact that mitochondrial proteins are encoded by both genomes.

In this study, we performed genome-wide analyses to assess mtSNP effects on BD risk, focusing on interaction effects between nuclear SNPs (nSNPs) and mtSNPs. We hypothesized that the impact of nuclear genetic variation on $\mathrm{BD}$ risk could be modified by the mtDNA that a person carries and perhaps more nuclear BD risk loci would be uncovered by incorporating mitochondrial interactions into the risk model. In addition, we also investigated a potential role of $\mathrm{mtSNP}$-nSNP interactions for earlyonset BD (EOBD) compared to later-onset cases. Given previous studies showing the significance of age of onset of $\mathrm{BD}$ as a predictor of familial risk (relatives of EOBD patients had significantly greater risks of BD than those of later-onset $\mathrm{BD}$ patients) [38], we hypothesized that by considering both the nuclear and the mitochondrial genomes additional genetic risk loci could be uncovered that influence age at BD onset.

\section{Methods and Materials}

\section{Study Description}

This study utilized publically available GWAS data from the GAIN BD study, which included 2,035 European American subjects (i.e., 1,001 BD cases [EOBD, $n=419$ ] and 1,034 controls) [39]. All BD cases met criteria for DSM-IV-defined bipolar I disorder, and controls were matched on age, sex, and ethnicity $[39,40]$. The age at $\mathrm{BD}$ onset (in years) was obtained from diagnostic interviews for the $\mathrm{BD}$ cases; $\mathrm{EOBD}$ was defined as a $\mathrm{BD}$ onset age of 19 years or younger [41]. Genotyping was performed using the Affymetrix 6.0 array that included nSNPs and mtSNPs. Basic quality control criteria were applied to exclude SNPs with a low minor allele frequency (MAF; $<10 \%$ ) and low call rates $(<95 \%)$, resulting in $544,209 \mathrm{nSNPs}$ and $15 \mathrm{mtSNPs}$ [42]. We chose a higher cutoff for MAF (i.e., $10 \%$ rather than a standard cutoff of $1-5 \%$ ), because this study focused on nSNP-mtSNP interaction effects, and the power to detect interactions declines more rapidly with decreasing MAF than does the power to detect main effects. A list of the $15 \mathrm{mtSNPs}$ included in our analyses with detailed annotation information is presented in online supplementary Table 1 (for all online suppl. material, see www.karger.com/doi/10.1159/000464444).

\section{Statistical Analysis}

For the nuclear genome, the effective number of independent SNPs was calculated to be used for Bonferroni multiple testing correction [43]. For the mitochondrial genome, the number of principal components (PCs) explaining at least $95 \%$ of the variability in mitochondrial genotypes was calculated. The total number of tests to be corrected for this study was then set to the effective number of independent nSNPs $(n=273,063)$ multiplied by the number of PCs from the mitochondrial genome (5 PCs). Therefore, association results with $\mathrm{p}<3.7 \times 10^{-8}(=273,063 \times 5)$ were considered statistically significant after multiple testing correction.

For each outcome (BD risk and EOBD among BD cases), logistic regression models were used to assess the joint effects of the nSNP main effect and the interaction effect between nSNP and $\mathrm{mtSNP}$, for each pair of mtSNP and nSNP, using 2df likelihood ratio tests. The $2 \mathrm{df}$ test jointly evaluates the effect of the last 2 terms (i.e., the nSNP effect and the nSNP $\times$ mtSNP interaction effect) from the following logistic regression model:

$\operatorname{Logit}(p)=\mathrm{a}_{0}+\mathrm{a}_{1} \times \mathrm{mtSNP}+\mathrm{a}_{2} \times \mathrm{nSNP}+\mathrm{a}_{3} \times \mathrm{nSNP} \times \mathrm{mtSNP}$,

where $p$ is the probability of the outcome (e.g., $\mathrm{BD}$ vs. control), and $a_{0} / a_{1} / a_{2} / a_{3}$ are regression parameters for terms included in the model. The rationale for this analysis is to jointly assess the effect of the nSNP main effect and/or the nSNP $\times$ mtSNP interaction term (i.e., testing if both $\mathrm{a}_{2}=0$ and $\mathrm{a}_{3}=0$ ), thereby testing the nSNP effect while allowing for modification of the effect by the mtSNP genotype. In addition, $1 \mathrm{df}$ likelihood ratio tests of the $\mathrm{nSNP} \times \mathrm{mtSNP}$ interaction terms were also performed (i.e., test assessing whether $\mathrm{a}_{3}=0$ ).

All models were further adjusted for the first PC from the nuclear genome (nPC1), and the interaction between $\mathrm{nPC1}$ and mtSNP to prevent potential confounding by population stratification. For mtSNPs showing the strongest associations based on the joint tests, genome-wide analyses were repeated using imputed SNP data to generate Manhattan plots and locus zoom plots. Genome-wide imputation for the GAIN BD data was conducted using the 1,000 Genome Project cosmopolitan reference panel, and the detailed procedures have been previously described [44].

In addition to SNP level analysis, gene set analyses were conducted using a competitive method implemented in MAGENTA [45] to test whether nSNPs in mitochondrion-related genes were more enriched for interactions with individual mtSNPs compared to those not related to mitochondria. A gene set analysis was performed for each outcome (risk of $\mathrm{BD}$ and $\mathrm{EOBD}$ ), comparing the mtSNP interaction results for nSNPs in 967 autosomal mitochondrial genes described in MitoCarta [28], with mtSNP interaction results for autosomal nSNPs outside of this set of 967 genes. Since both outcomes are considered highly polygenic, a gene set enrichment analysis (GSEA) cutoff using the top $25 \%$ of gene level statistics, recommended by the authors of MAGENTA, was used for comparison between the 967 genes in MitoCarta and those not in the MitoCarta list. Gene boundaries were based on the provided build 37 locations in the MAGENTA software with a 40-kb downstream and 110-kb upstream buffer for each gene. The HLA genes were removed as suggested by the authors of MAGENTA. GSEA $p$ values were computed via permutation, with a minimum of 10,000 and a maximum of $1,000,000$ permutations. 
Table 1. Top 3 association results based on $2 \mathrm{df}$ tests for risk of bipolar disorder, and 2 subsequent association results for each of the top mtSNPs

\begin{tabular}{|c|c|c|c|c|c|c|c|c|c|c|c|c|c|}
\hline \multicolumn{5}{|l|}{ mtSNP } & \multicolumn{5}{|l|}{ nSNP } & \multicolumn{4}{|c|}{ Association results } \\
\hline rs28358279 & 10,463 & tRNA & G & 0.11 & $\begin{array}{l}\text { rs1124376 } \\
\text { rs10000984 } \\
\text { rs11764581 }\end{array}$ & $\begin{array}{l}\text { 3: } 20108546 \\
\text { 4: } 175644564 \\
7: 141712467\end{array}$ & $\begin{array}{l}\text { PCAF/KAT2B } \\
\text { GLRA3 } \\
\text { MGAM }\end{array}$ & $\begin{array}{l}\text { A } \\
\text { G } \\
\text { C }\end{array}$ & $\begin{array}{l}0.22 \\
0.44 \\
0.13\end{array}$ & $\begin{array}{l}2.8 \times 10^{-6} \\
2.8 \times 10^{-6} \\
7.0 \times 10^{-6}\end{array}$ & $\begin{array}{l}5.0 \times 10^{-5} \\
9.8 \times 10^{-7} \\
6.7 \times 10^{-4}\end{array}$ & $\begin{array}{l}0.87 \\
0.97 \\
1.25\end{array}$ & $\begin{array}{l}0.26 \\
3.06 \\
3.85\end{array}$ \\
\hline
\end{tabular}

MtSNP-nSNP interaction test results are also shown for the same SNP pairs. mtSNP, mitochondrial single-nucleotide polymorphism; nSNP, nuclear single-nucleotide polymorphism; SNP, single-nucleotide polymorphism; BP, base pair; MA, minor allele; MAF, minor allele frequency; Chr, chromosome. ${ }^{1}$ In the presence of a major mtSNP allele, OR for adding 1 copy of an nSNP minor allele based on the interaction analysis. ${ }^{2}$ In the presence of a minor mtSNP allele, OR for adding 1 copy of an nSNP minor allele based on the interaction analysis.

Table 2. Top 3 association results based on $2 \mathrm{df}$ tests for risk of early-onset bipolar disorder, and 2 subsequent association results for each of the top mtSNPs

\begin{tabular}{|c|c|c|c|c|c|c|c|c|c|c|c|c|c|}
\hline \multicolumn{5}{|l|}{ mtSNP } & \multicolumn{5}{|l|}{ nSNP } & \multicolumn{4}{|c|}{ Association results } \\
\hline rs3928306 & 3,010 & RNR2 & A & 0.24 & $\begin{array}{l}\text { rs } 2420932 \\
\text { rs6597183 } \\
\text { rs7987059 }\end{array}$ & $\begin{array}{c}10: 123118218 \\
6: 6042429 \\
13: 27541625\end{array}$ & $\begin{array}{l}\text { FGFR2 } \\
\text { NRN1 } \\
\text { LOC100129306 }\end{array}$ & $\begin{array}{l}\mathrm{G} \\
\mathrm{T} \\
\mathrm{T}\end{array}$ & $\begin{array}{l}0.18 \\
0.22 \\
0.19\end{array}$ & $\begin{array}{l}4.5 \times 10^{-7} \\
1.4 \times 10^{-6} \\
1.2 \times 10^{-5}\end{array}$ & $\begin{array}{l}9.4 \times 10^{-8} \\
2.1 \times 10^{-5} \\
2.5 \times 10^{-6}\end{array}$ & $\begin{array}{l}1.29 \\
1.07 \\
0.66\end{array}$ & $\begin{array}{l}0.20 \\
1.07 \\
0.65\end{array}$ \\
\hline
\end{tabular}

MtSNP-nSNP interaction test results are also shown for the same SNP pairs. mtSNP, mitochondrial single-nucleotide polymorphism; nSNP, nuclear single-nucleotide polymorphism; SNP, single-nucleotide polymorphism; BP, base pair; MA, minor allele; MAF, minor allele frequency; Chr, chromosome. ${ }^{1}$ In the presence of a major mtSNP allele, OR for adding 1 copy of an nSNP minor allele. ${ }^{2}$ In the presence of a minor mtSNP allele, OR for adding 1 copy of an nSNP minor allele.

\section{Results}

None of the association results from the $2 \mathrm{df}$ tests or the $1 \mathrm{df}$ interaction tests passed the stringent significance threshold corrected for multiple testing $\left(p<3.7 \times 10^{-8}\right)$. Tables 1 and 2 present the top 3 association signals based on the $2 \mathrm{df}$ test for the analyses of $\mathrm{BD}$ risk and $\mathrm{EOBD}$, respectively; Tables 1 and 2 also include 2 subsequent association results for each of the 3 top mtSNPs. In Tables 1 and 2 we report top findings based on the joint effect (2df test), while not including any results with $p>0.001$ for the $\mathrm{nSNP} \times \mathrm{mtSNP}$ interaction effect. We only present joint effect test results for pairs of SNP with at least "marginal" evidence of an interaction effect (defined as $p_{\text {int }} \leq$ 0.001 ), because the association results for $n S N P-m t S N P$ pairs with little evidence for an interaction effect $\left(p_{\text {int }}>\right.$ 0.001 ) are mainly driven by the nSNP main effects that have been extensively tested in prior GWAS studies and are not the focus of this study. 


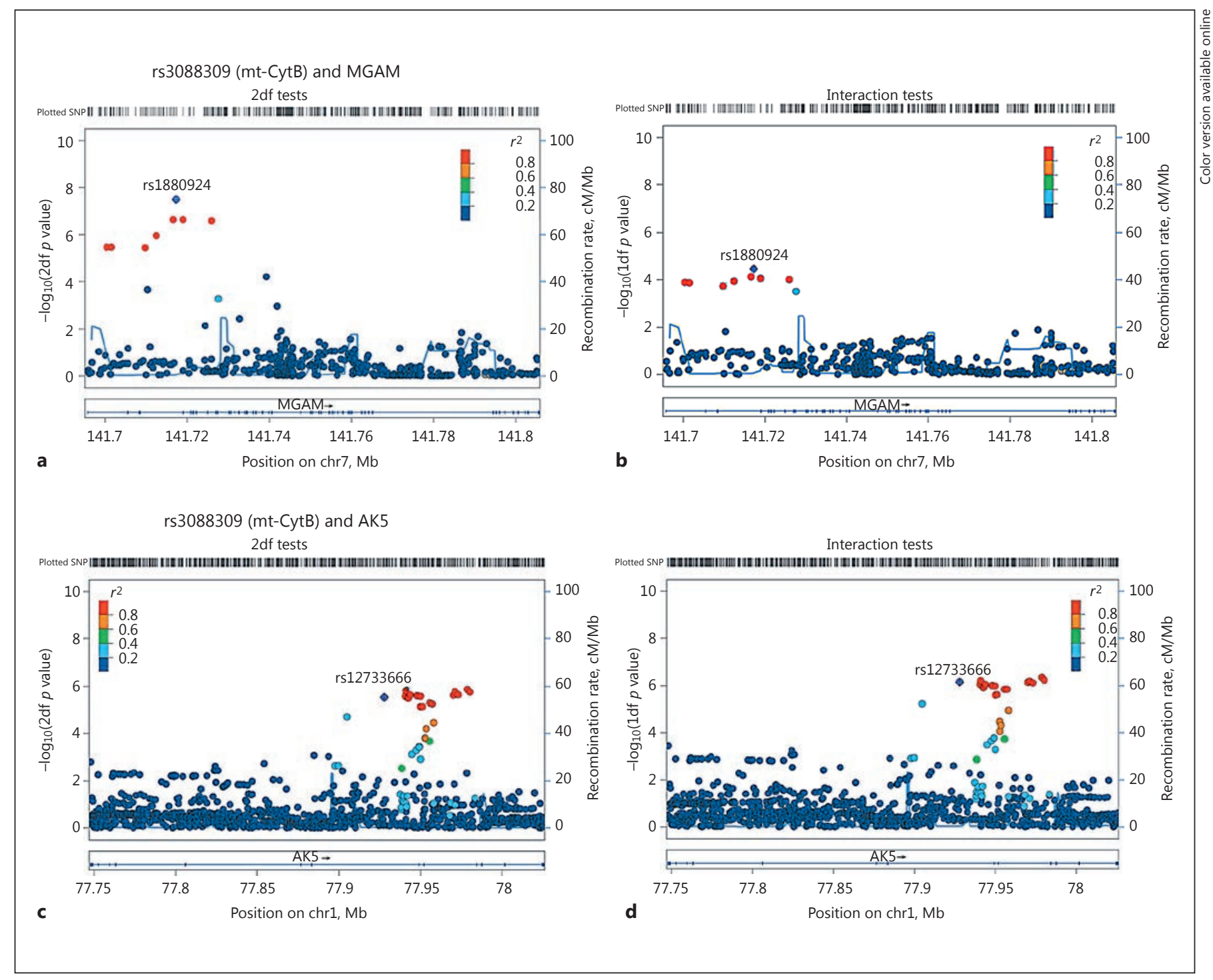

Fig. 1. Regional association plots for the top 2 gene regions (MGAM and AK5) with rs3088309 (Mt-CytB) for risk of bipolar disorder. a, $\mathbf{c} p$ values from $2 \mathrm{df}$ tests. b, d Interaction test $p$ values. chr7, chromosome 7; chr1, chromosome 1; SNP, single-nucleotide polymorphism.

The strongest evidence of an association with $\mathrm{BD}$ risk was observed for mtSNP rs3088309 (MAF $=0.21$ for allele T) located in the mitochondrial encoded cytochrome $\mathrm{B}(C y t B)$ gene and the nSNP rs1880924 (MAF $=0.14$ for allele A) located in the maltase-glucoamylase (MGAM) on chromosome 7, a gene involved in glycosaminoglycan metabolism pathways $\left(p_{2 \mathrm{df}}=8.2 \times 10^{-8}\right.$; Table 1 ; Fig. 1; online suppl. Fig. 1). The interaction between these variants showed a moderate level of association with $\mathrm{BD}$ risk (Table 1 ; $p_{\text {int }}=1.4 \times 10^{-4}$ ), implying the joint test signal is partially due to the nSNP main effect and partially due to the nSNP-mtSNP interaction effect supporting the epistatic interaction between the nuclear and mitochondrial genes. The odds ratio (OR) for one copy of the minor nSNP allele was 3.42 among carriers of the minor allele A of the mtSNP and 1.27 among carriers of the common allele $\mathrm{G}$ of the mtSNP (Fig. 3a). Among other top association results involving the same $\mathrm{mtSNP}$ (rs3088309), the joint effect with rs12733666 located in adenylate kinase 5 (AK5) showed a trend for significance $\left(p_{2 \mathrm{df}}=2.9 \times 10^{-6}\right)$, with this joint test result being entirely driven by the nSNP-mtSNP interaction 


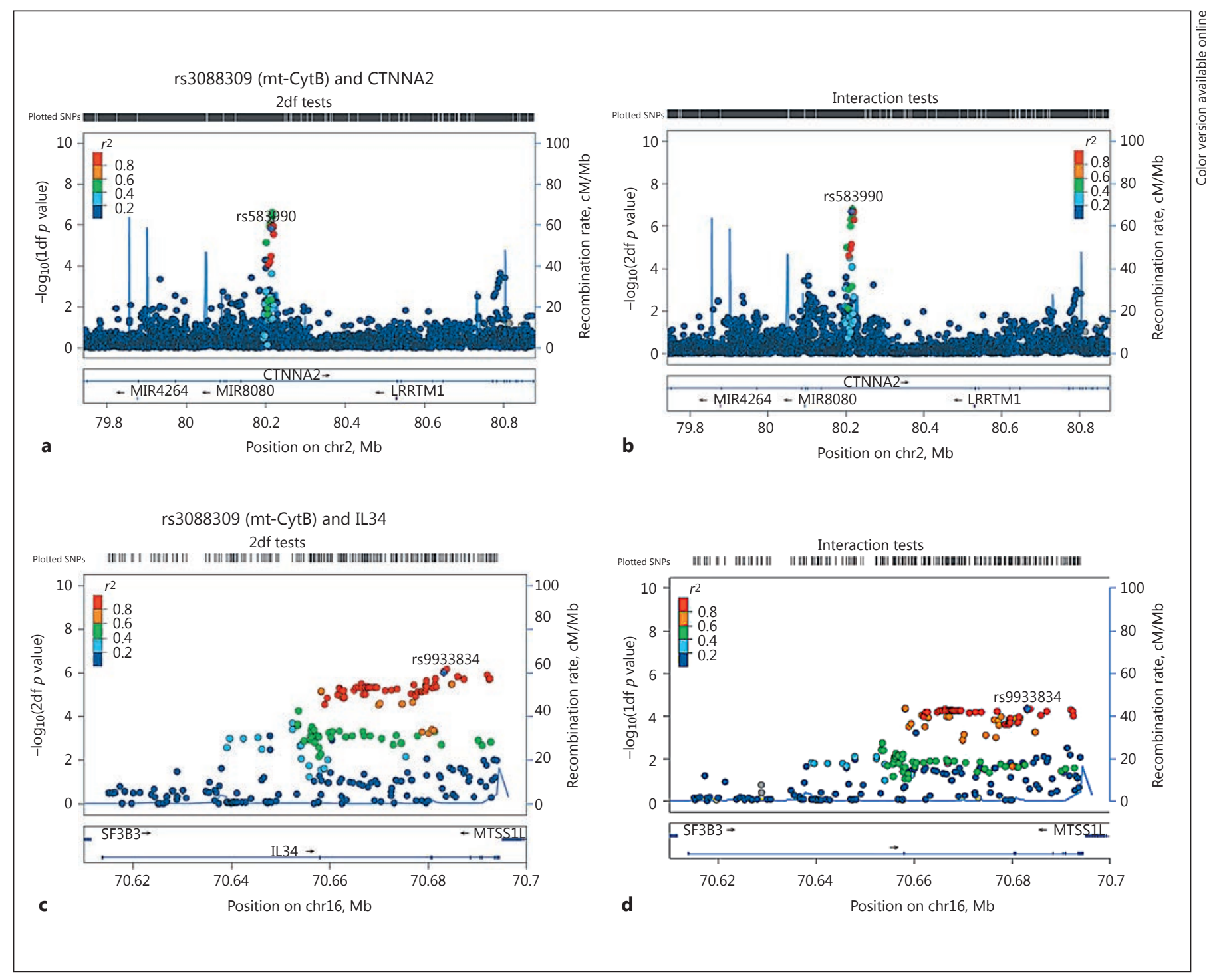

Fig. 2. Regional association plots for top 2 gene regions (CTNNA2 and IL34) with rs3088309 (Mt-CytB) for the risk of early-onset bipolar disorder. a, $\mathbf{c} p$ values from $2 \mathrm{df}$ tests. b, d Interaction test $p$ values. chr2, chromosome 2; chr16, chromosome 16; SNP, single-nucleotide polymorphism.

$\left(p_{\text {int }}=7.1 \times 10^{-7}\right)$. The second most significant association was observed with rs3915952 located in Mt-ND4 and nSNP rs1880924, the same MGAM SNP from the top association result discussed above. Rs3915952 is highly correlated with the top mtSNP rs3088309 $\left(r^{2}=\right.$ 0.95), implying that the second association signal is not independent of the top hit. In fact, both mtSNPs rs3088309 and rs3915952 are part of mitochondrial haplogroup JT defining SNP.

For EOBD risk among $\mathrm{BD}$ patients, the top association signal was with the mtSNP rs3088309 in CytB (the same
$\mathrm{mtSNP}$ that is involved in the top BD risk association), and the nSNP rs583990 (MAF $=0.25$ for allele A) located in the Cadherin-associated protein, Alpha2 (CTNNA2) on chromosome $2\left(p_{2 \mathrm{df}}=2.1 \times 10^{-7}\right.$ and $\left.p_{\text {int }}=1.6 \times 10^{-6}\right)$. The results suggest that among BD patients the minor allele A of nSNP rs583990 is associated with a greater risk of EOBD (i.e., a higher risk of developing BD by the age of 19 years), and the effect of the nSNP is greater for carriers of the minor $\mathrm{mtSNP}$ rs3088309 $\mathrm{T}$ allele, compared to those with the major $\mathrm{mtSNP}$ allele $(\mathrm{OR}=4.53$ vs. 1.05 ; Fig. 3b). The mtSNP rs3088309 also has suggestive evi- 
Fig. 3. OR for each combination of $\mathrm{mtSNP}$ rs3088309 (0/1 for the minor allele) and nSNP $(0 / 1 / 2$ for the number of minor alleles) genotypes for top 2 association results from $2 \mathrm{df}$ tests for the risk of bipolar disorder (a) and early-onset bipolar disor$\operatorname{der}(\mathbf{b})$. The pair $(\mathrm{mtSNP}=0$ and $\mathrm{nSNP}=$ 0 ) was used as the reference category (i.e., $\mathrm{OR}=1$ ). mtSNP, mitochondrial single-nucleotide polymorphism; nSNP, nuclear single-nucleotide polymorphism.

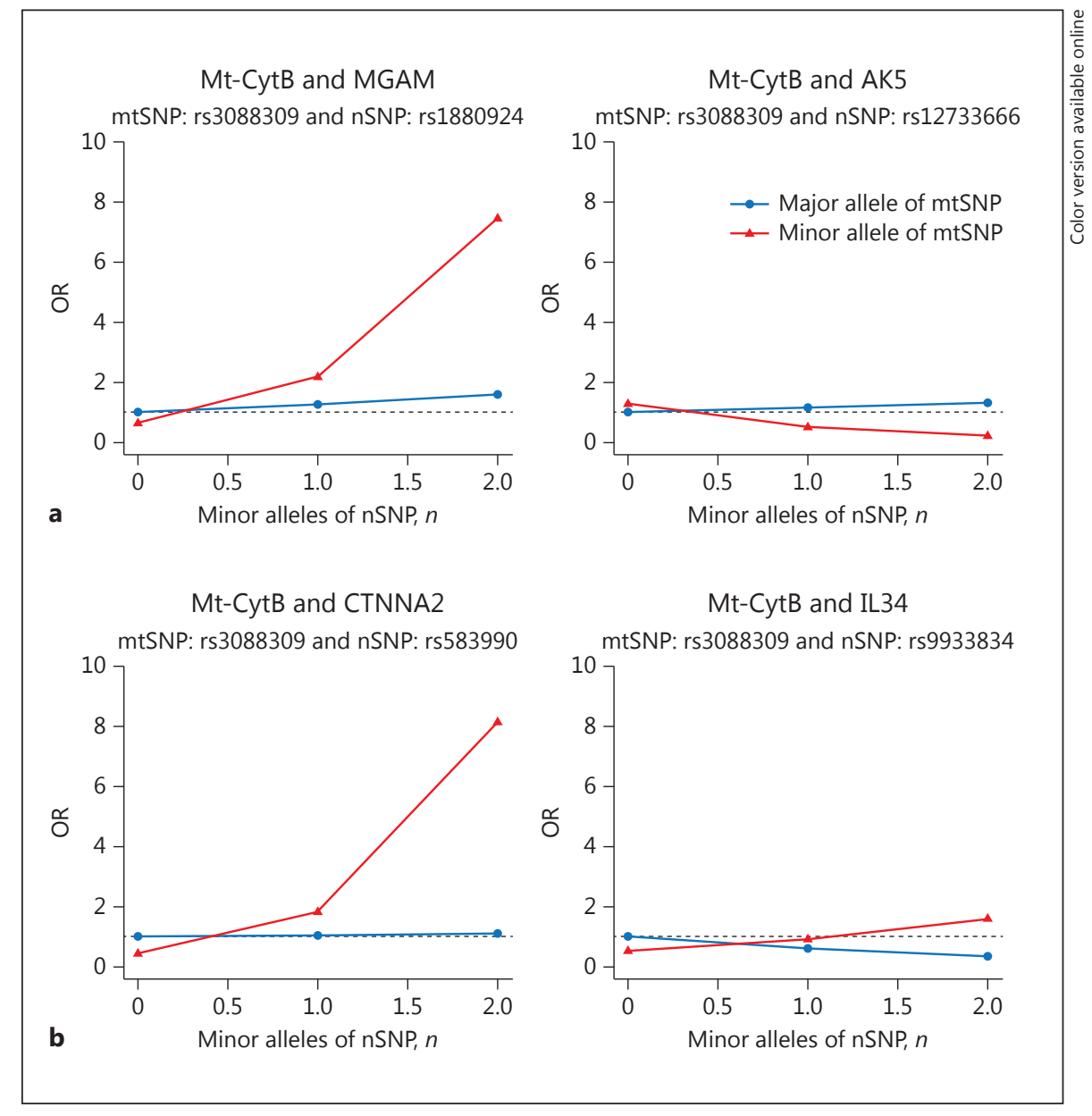

dence for interacting with nSNP rs9933834 located in interleukin $34\left(I L 34 ; p_{\text {joint }}=1.1 \times 10^{-6}\right.$; interaction $p_{\text {int }}=1.6$ $\times 10^{-5}$ ) for the risk of EOBD (Table 2; Fig. 2b, 3b). The second highest association signal involved the mtSNP rs3915952 in ND4 and rs9933834 in IL34 on chromosome 16 (Table 2; Fig. 2; online suppl. Fig. 2).

When testing for enrichment of nSNP-mtSNP interaction association, no significant enrichment was found for the 967 mitochondrial-related genes (online suppl. Table 2). The highest evidence of enrichment was observed for mtSNP rs28359178 located in ND5 for risk of BD (240 for the observed number of genes above the cutoff compared to 217 expected genes; GSEA $p=0.05$ ), and with rs3899498 (located in ND5; GSEA $p=0.05$ ) and rs28357682 (located in $C y t B$; GSEA $p=0.05$ ) for risk of EOBD. These gene set analysis results are not significant after correction for multiple testing incurred by performing an enrichment analysis for each of $15 \mathrm{mtSNPs}$ with an MAF $>0.10$.

MtSNP Contribution to BD

\section{Discussion}

To our knowledge, this is the first GWAS to investigate $\mathrm{mtSNP}$ contribution in $\mathrm{BD}$, focusing on $\mathrm{nSNP}-\mathrm{mtSNP}$ epistatic interaction and the risk of $\mathrm{BD}$ as well as EOBD (a $\mathrm{BD}$ subphenotype). Although no association results were genome-wide significant, some highly notable associations were detected when accounting for mtSNP interaction effects, including nuclear genes that were previously reported BD susceptibility genes or have been implicated in neuropathology. This proof-of-concept study suggests the potential importance of investigating both nuclear and mitochondrial genomes in studying the complex genetic predisposition for $\mathrm{BD}$ as well as the age of disease onset. By modeling the modifying effects of mtSNPs on the contribution of nSNPs to BD risk and age of disease onset, additional genetic factors may be identified.

Mt-CytB SNP rs3088309 (and the highly correlated $\mathrm{mtSNP}$ rs3915952 in ND4) variants were the top mtSNP 
with suggestive evidence of interaction with nSNPs in contributing to $\mathrm{BD}$ risk and $\mathrm{EOBD}$. The $\mathrm{Mt}-\mathrm{CytB}$ gene is responsible for making cytochrome $\mathrm{B}$, which is a key protein for transferring the electrons (carrier), and cytochrome $\mathrm{B}$ is the only component of complex III that is produced by mtDNA [46]. The mtSNP rs3088309 (merged into rs527236209) located in the Mt-CytB gene, is a missense variant that causes an amino acid change from leucine (major allele) to isoleucine (minor allele) at position 236. Given the relevance of the Mt-CytB gene to the electron transport chain, any mutation in this gene potentially contributes reactive oxygen species, which has been shown to be elevated in BD [14, 15]. Being essential for electron transport chain, the mutations in $\mathrm{CytB}$ rs3088309 and ND4 rs3915952 might leave neurons more vulnerable to genetic and environmental risk factors. In addition, mitochondrial haplogroup JT (the top mtSNPs rs3088309 and rs3915952 are part of the haplogroup-defining SNP) has been implicated in early-onset schizophrenia, though the mechanism underlying this association is still unknown [47].

The results of this study suggest that for the risk of $B D$ the effects of nuclear genes $M G A M$ and $A K 5$ are modulated by variants in mitochondrial genes. $A K 5$ is mainly expressed in the brain and it has been implicated in temporal lobe epilepsy, autoimmune limbic encephalitis, and Parkinson's and Alzheimer's diseases [48-51]. Our results suggest that carrying the minor allele of $A K 5$ rs12733666 may have a protective effect for BD in those carrying the minor $\mathrm{mtSNP}$ allele. The MGAM gene, related to starch metabolism, has been shown to be downregulated by ZNF804A overexpression (a gene implicated in susceptibility to schizophrenia) [52]. In addition, subjects with autism have dysregulated mRNA expression of MGAM [53]. Considering its central role in starch metabolism [54], it is plausible that genetic variation in MGAM coupled with particular mutations in the mitochondrial genome may result in mitochondrial dysfunction, which may have significant implications for $\mathrm{BD}$ pathophysiology.

Our results also suggest that nuclear genes CTNNA2 and IL34 may interact with mitochondrial variants in modulating the risk of EOBD. CTNNA2 is highly expressed in the brain and encodes an $\alpha$-catenin important for synaptic contact and neuronal plasticity. A CTNNA2 polymorphism has been implicated in a highly heritable excitement-seeking trait, a common trait in BD [55]. CTNNA2 knockout mice show axon migration defects as well as hippocampal and cerebellar lamination defects that are associated with impairments in startle responses and fear conditioning $[56,57]$. Furthermore, a CTNNA2 SNP was associated with BD in a GWAS study [58]. IL34 is a cytokine that is implicated in immune response and might play an important role in inflammatory mechanisms in mood disorders $[59,60]$. For instance, IL34 cytokine is important for the survival of microglia, the macrophages of the brain that determine the levels of inflammation in the cellular environments [61]. It has been suggested that mitochondrial dysfunction may be induced by excessive reactive oxygen species produced by activated microglia [62].

This work demonstrates a novel approach to studying genetic effects on $\mathrm{BD}$; however, the results must be interpreted in light of the limitations of this study. Detection of interaction effects requires a significantly larger sample size compared to standard main-effect genetic analysis. This study was based on a small sample, which limited the power to detect genome-wide significant associations; to partially overcome challenges related to power in this study we limited the analyses to common SNPs with an MAF $>0.1$. While none of the mtSNP-nSNP interactions were significant after multiple testing correction, replication of the top interaction signals would strengthen the presented findings. A further limitation is that this study used mtSNP genotypes from blood samples, not from brain samples. However, although mtDNA accumulates different mutations in different tissues leading to heteroplasmy differences between tissues, previous research has demonstrated perfect concordance between homoplasmic variants (such as mtSNPs used in this study) in 11 brain regions and blood, suggesting that blood is suitable for the study of homoplasmic mtDNA variation [37].

Although none of the associations were statistically significant after multiple testing correction, this study is the first GWAS investigating the effects of mitochondrial genome variation on the risk of $\mathrm{BD}$ and a $\mathrm{BD}$ subphenotype (early onset vs. adult onset), while considering the combined effects of nuclear and mitochondrial genetic variants. Our novel approach of investigating mtSNP modulation of the nSNP contribution to the risk of $\mathrm{BD}$ found suggestive evidence of the joint effect of mtSNPs and several candidate genes with biological relevance to $\mathrm{BD}$ and its onset. This concept of analyzing nSNP effects while accounting for interactions with mtSNPs warrants further investigation in larger samples.

In conclusion, our study demonstrated the potential importance of considering the mitochondrial genome for uncovering additional genetic factors in $\mathrm{BD}$, especially for nuclear genes that do not have a strong signal alone but whose effects depend on mitochondrial genetic variants. 


\section{Acknowledgements}

This study was funded by the Marriot Foundation, the Mayo

Clinic Center for Individualized Medicine, and a small grant award supported by the Department of Health Sciences Research, Mayo Clinic.

\section{Disclosure Statement}

The authors have nothing to disclose.

\section{References}

1 Frye MA: Clinical practice: bipolar disorder - a focus on depression. N Engl J Med 2011; 364:51-59.

2 American Psychiatric Association: Diagnostic and Statistical Manual of Mental Disorders, ed 5. Arlington, APA, 2013.

3 Collins PY, Patel V, Joestl SS, March D, Insel TR, Daar AS, Anderson W, Dhansay MA, Phillips A, Shurin S, et al: Grand challenges in global mental health. Nature 2011;475:27-30.

4 Shinozaki G, Potash JB: New developments in the genetics of bipolar disorder. Curr Psychiatry Rep 2014;16:493.

5 Ou X, Crane DE, MacIntosh BJ, Young LT, Arnold P, Ameis S, Goldstein BI: CACNA1C rs1006737 genotype and bipolar disorder: focus on intermediate phenotypes and cardiovascular comorbidity. Neurosci Biobehav Rev 2015;55:198-210.

6 Gershon ES, Alliey-Rodriguez N, Liu C: After GWAS: searching for genetic risk for schizophrenia and bipolar disorder. Am J Psychiatry 2011;168:253-256.

7 de Sousa RT, Machado-Vieira R, Zarate CA Jr, Manji HK: Targeting mitochondrially mediated plasticity to develop improved therapeutics for bipolar disorder. Expert Opin Ther Targets 2014;18:1131-1147.

8 Clay HB, Sillivan S, Konradi C: Mitochondrial dysfunction and pathology in bipolar disorder and schizophrenia. Int J Dev Neurosci 2011;29:311-324.

9 Sequeira A, Rollins B, Magnan C, van Oven M, Baldi P, Myers RM, Barchas JD, Schatzberg AF, Watson SJ, Akil H, et al: Mitochondrial mutations in subjects with psychiatric disorders. PLoS One 2015;10:e127280.

10 Andreazza AC, Young LT: The neurobiology of bipolar disorder: identifying targets for specific agents and synergies for combination treatment. Int J Neuropsychopharmacol 2014;17:1039-1052.

11 Andreazza AC, Frey BN, Erdtmann B, Salvador M, Rombaldi F, Santin A, Goncalves CA, Kapczinski F: DNA damage in bipolar disorder. Psychiatry Res 2007;153:27-32.

12 Scola G, Kim HK, Young LT, Andreazza AC: A fresh look at complex I in microarray data: clues to understanding disease-specific mitochondrial alterations in bipolar disorder. Biol Psychiatry 2013;73:e4-e5.

13 Konradi C, Eaton M, MacDonald ML, Walsh J, Benes FM, Heckers S: Molecular evidence for mitochondrial dysfunction in bipolar disorder. Arch Gen Psychiatry 2004;61:300-308.
14 Andreazza AC, Wang JF, Salmasi F, Shao L, Young LT: Specific subcellular changes in oxidative stress in prefrontal cortex from patients with bipolar disorder. J Neurochem 2013;127:552-561.

15 Andreazza AC, Shao L, Wang JF, Young LT: Mitochondrial complex I activity and oxidative damage to mitochondrial proteins in the prefrontal cortex of patients with bipolar disorder. Arch Gen Psychiatry 2010;67:360-368.

16 Kim HK, Mendonca KM, Howson PA Brotchie JM, Andreazza AC: The link between mitochondrial complex I and brain-derived neurotrophic factor in SH-SY5Y cells the potential of JNX1001 as a therapeutic agent. Eur J Pharmacol 2015;764:379-384.

17 Anglin RE, Garside SL, Tarnopolsky MA, Mazurek MF, Rosebush PI: The psychiatric manifestations of mitochondrial disorders: a case and review of the literature. J Clin Psychiatry 2012;73:506-512.

18 Mertens J, Wang QW, Kim Y, Yu DX, Pham S, Yang B, Zheng Y, Diffenderfer KE, Zhang J, Soltani S, et al: Differential responses to lithium in hyperexcitable neurons from patients with bipolar disorder. Nature 2015;527:95-99.

19 Munakata K, Tanaka M, Mori K, Washizuka S, Yoneda M, Tajima O, Akiyama T, Nanko S, Kunugi H, Tadokoro K, et al: Mitochondrial DNA 3644T $->$ C mutation associated with bipolar disorder. Genomics 2004;84:10411050.

20 Chang CC, Jou SH, Lin TT, Liu CS: Mitochondrial DNA variation and increased oxidative damage in euthymic patients with bipolar disorder. Psychiatry Clin Neurosci 2014;68:551-557.

21 Kato T, Takahashi Y: Deletion of leukocyte mitochondrial DNA in bipolar disorder. J Affect Disord 1996;37:67-73.

22 Kazuno AA, Munakata K, Mori K, Nanko S, Kunugi H, Nakamura K, Mori N, Yamada K, Yoshikawa T, Kato N, et al: Mitochondrial DNA haplogroup analysis in patients with bipolar disorder. Am J Med Genet B Neuropsychiatr Genet 2009;150B:243-247.

23 Washizuka S, Ikeda A, Kato N, Kato T: Possible relationship between mitochondrial DNA polymorphisms and lithium response in bipolar disorder. Int J Neuropsychopharmacol 2003;6:421-424.

24 Kato T, Kunugi H, Nanko S, Kato N: Association of bipolar disorder with the 5178 polymorphism in mitochondrial DNA. Am J Med Genet 2000;96:182-186.
25 Li CT, Bai YM, Hsieh JC, Lee HC, Yang BH, Chen MH, Lin WC, Tsai CF, Tu PC, Wang SJ, et al: Peripheral and central glucose utilizations modulated by mitochondrial DNA 10398A in bipolar disorder. Psychoneuroendocrinology 2015;55:72-80.

26 Kato T: The other, forgotten genome: mitochondrial DNA and mental disorders. Mol Psychiatry 2001;6:625-633.

27 Kakiuchi C, Ishiwata M, Kametani M, Nelson C, Iwamoto K, Kato T: Quantitative analysis of mitochondrial DNA deletions in the brains of patients with bipolar disorder and schizophrenia. Int J Neuropsychopharmacol 2005;8: 515-522.

28 Calvo SE, Clauser KR, Mootha VK: MitoCarta2.0: an updated inventory of mammalian mitochondrial proteins. Nucleic Acids Res 2016;44:D1251-D1257.

29 Chubb JE, Bradshaw NJ, Soares DC, Porteous DJ, Millar JK: The DISC locus in psychiatric illness. Mol Psychiatr 2008;13:36-64.

30 Wang Q, Charych EI, Pulito VL, Lee JB, Graziane NM, Crozier RA, Revilla-Sanchez R, Kelly MP, Dunlop AJ, Murdoch H, et al: The psychiatric disease risk factors DISC1 and TNIK interact to regulate synapse composition and function. Mol Psychiatr 2011;16:1006-1023.

31 Xu C, Li PP, Kennedy JL, Green M, Hughes B, Cooke RG, Parikh SV, Warsh JJ: Further support for association of the mitochondrial complex I subunit gene NDUFV2 with bipolar disorder. Bipolar Disord 2008;10:105-110.

32 Doyle GA, Dahl JP, Bloch PJ, Weller AE, Lohoff FW, Ferraro TN, Berrettini WH: Association study of polymorphisms in the autosomal mitochondrial complex I subunit gene, $\mathrm{NADH}$ dehydrogenase (ubiquinone) flavoprotein 2, and bipolar disorder. Psychiatr Genet 2011;21:51-52.

33 Washizuka S, Iwamoto K, Kakiuchi C, Bundo M, Kato T: Expression of mitochondrial complex I subunit gene NDUFV2 in the lymphoblastoid cells derived from patients with bipolar disorder and schizophrenia. Neurosci Res 2009;63:199-204.

34 Rosa A, Abrantes P, Sousa I, Francisco V, Santos P, Francisco D, Xavier JM, Oliveira SA: Ulcerative colitis is under dual (mitochondrial and nuclear) genetic control. Inflamm Bowel Dis 2016;22:774-781.

35 Bulstrode H, Nicoll JA, Hudson G, Chinnery PF, Di Pietro V, Belli A: Mitochondrial DNA and traumatic brain injury. Ann Neurol 2014; 75:186-195. 
36 Maruszak A, Safranow K, Branicki W, Gaweda-Walerych K, Pospiech E, Gabryelewicz T, Canter JA, Barcikowska M, Zekanowski C: The impact of mitochondrial and nuclear DNA variants on late-onset Alzheimer's disease risk. J Alzheimers Dis 2011;27:197-210.

37 Sequeira A, Martin MV, Rollins B, Moon EA, Bunney WE, Macciardi F, Lupoli S, Smith EN, Kelsoe J, Magnan CN, et al: Mitochondrial mutations and polymorphisms in psychiatric disorders. Front Genet 2012;3:103.

38 Somanath CP, Jain S, Reddy YC: A family study of early-onset bipolar I disorder. J Affect Disord 2002;70:91-94.

39 Smith EN, Bloss CS, Badner JA, Barrett T, Belmonte PL, Berrettini W, Byerley W, Coryell W, Craig D, Edenberg HJ, et al: Genome-wide association study of bipolar disorder in European American and African American individuals. Mol Psychiatr 2009;14:755-763.

40 Winham SJ, Cuellar-Barboza AB, Oliveros A, McElroy SL, Crow S, Colby C, Choi DS, Chauhan M, Frye M, Biernacka JM: Genomewide association study of bipolar disorder accounting for effect of body mass index identifies a new risk allele in TCF7L2. Mol Psychiatr 2014;19:1010-1016.

41 Mahon PB, Pirooznia M, Goes FS, Seifuddin F, Steele J, Lee PH, Huang J, Hamshere ML, DePaulo JR, Kelsoe JR, et al: Genome-wide association analysis of age at onset and psychotic symptoms in bipolar disorder. Am J Med Genet B Neuropsychiatr Genet 2011; 156B:370-378.

42 Jacobsen KK, Nievergelt CM, Zayats T, Greenwood TA, Anttila V, Akiskal HS, Haavik J, Fasmer OB, Kelsoe JR, Johansson S, et al: Genome wide association study identifies variants in NBEA associated with migraine in bipolar disorder. J Affect Disord 2015;172:453-461.

43 Hendricks AE, Dupuis J, Logue MW, Myers RH, Lunetta KL: Correction for multiple testing in a gene region. Eur J Hum Genet 2014; 22:414-418.

44 Winham SJ, Cuellar-Barboza AB, McElroy SL, Oliveros A, Crow S, Colby CL, Choi DS, Chauhan M, Frye MA, Biernacka JM: Bipolar disorder with comorbid binge eating history: a genome-wide association study implicates APOB. J Affect Disord 2014;165:151-158.

45 Segre AV, Groop L, Mootha VK, Daly MJ, Altshuler D, DIAGRAM Consortium; MAGIC Investigators: Common inherited variation in mitochondrial ghenes is not enriched for associations with type 2 diabetes or related glycemic traits. PLoS Genet 2010;6:e1001058.

46 Shao L, Martin MV, Watson SJ, Schatzberg A, Akil H, Myers RM, Jones EG, Bunney WE, Vawter MP: Mitochondrial involvement in psychiatric disorders. Ann Med 2008;40:281295.

47 Magri C, Gardella R, Barlati SD, Valsecchi P, Sacchetti E, Barlati S: Mitochondrial DNA haplogroups and age at onset of schizophrenia. Am J Med Genet B Neuropsychiatr Genet 2007;144B:496-501.

48 Lai Y, Hu X, Chen G, Wang X, Zhu B: Downregulation of adenylate kinase 5 in temporal lobe epilepsy patients and rat model. J Neurol Sci 2016;366:20-26.

$49 \mathrm{Ng}$ AS, Kramer J, Centurion A, Dalmau J, Huang E, Cotter JA, Geschwind MD: Clinicopathological correlation in adenylate kinase 5 autoimmune limbic encephalitis. J Neuroimmunol 2015;287:31-35.

50 Garcia-Esparcia P, Hernandez-Ortega K, Ansoleaga B, Carmona M, Ferrer I: Purine metabolism gene deregulation in Parkinson's disease. Neuropathol Appl Neurobiol 2015; 41:926-940.

51 Ansoleaga B, Jove M, Schluter A, Garcia-Esparcia P, Moreno J, Pujol A, Pamplona R, Portero-Otin M, Ferrer I: Deregulation of purine metabolism in Alzheimer's disease. Neurobiol Aging 2015;36:68-80.

52 Umeda-Yano S, Hashimoto R, Yamamori H, Okada T, Yasuda Y, Ohi K, Fukumoto M, Ito A, Takeda M: The regulation of gene expression involved in TGF-beta signaling by ZNF804A, a risk gene for schizophrenia. Schizophr Res 2013;146:273-278.

53 Williams BL, Hornig M, Buie T, Bauman ML, Cho Paik M, Wick I, Bennett A, Jabado O, Hirschberg DL, Lipkin WI: Impaired carbohydrate digestion and transport and mucosal dysbiosis in the intestines of children with autism and gastrointestinal disturbances. PLoS One $2011 ; 6: \mathrm{e} 24585$.

54 Xiang R, Oddy VH, Archibald AL, Vercoe PE, Dalrymple BP: Epithelial, metabolic and innate immunity transcriptomic signatures differentiating the rumen from other sheep and mammalian gastrointestinal tract tissues. Peer J 2016;4:e1762.

55 Terracciano A, Esko T, Sutin AR, de Moor MH, Meirelles O, Zhu G, Tanaka T, Giegling I, Nutile T, Realo A, et al: Meta-analysis of genome-wide association studies identifies common variants in CTNNA2 associated with excitement-seeking. Transl Psychiatry 2011;1:e49.

56 Park C, Falls W, Finger JH, Longo-Guess CM, Ackerman SL: Deletion in Catna2, encoding alpha N-catenin, causes cerebellar and hippocampal lamination defects and impaired startle modulation. Nat Genet 2002;31:279-284.

57 Uemura M, Takeichi M: Alpha N-catenin deficiency causes defects in axon migration and nuclear organization in restricted regions of the mouse brain. Dev Dyn 2006;235:25592566.

58 Scott LJ, Muglia P, Kong XQ, Guan W, Flickinger M, Upmanyu R, Tozzi F, Li JZ, Burmeister M, Absher D, et al: Genome-wide association and meta-analysis of bipolar disorder in individuals of European ancestry. Proc Natl Acad Sci USA 2009;106:7501-7506.

59 Lin $\mathrm{H}$, Lee E, Hestir K, Leo C, Huang M, Bosch E, Halenbeck R, Wu G, Zhou A, Behrens $\mathrm{D}$, et al: Discovery of a cytokine and its receptor by functional screening of the extracellular proteome. Science 2008;320:807-811.

60 Wohleb ES, Franklin T, Iwata M, Duman RS: Integrating neuroimmune systems in the neurobiology of depression. Nat Rev Neurosci 2016;17:497-511.

61 Hodes GE, Kana V, Menard C, Merad M, Russo SJ: Neuroimmune mechanisms of depression. Nat Neurosci 2015;18:1386-1393.

62 Witte ME, Mahad DJ, Lassmann H, van Horssen J: Mitochondrial dysfunction contributes to neurodegeneration in multiple sclerosis. Trends Mol Med 2014;20:179-187. 